Check for updates

The BMJ

Cite this as: $B M J 2021 ; 375: n 3151$ http://dx.doi.org/10.1136/bmi.n3151 Published: 24 December 2021

\title{
Covid-19: Hospital admission $50-70 \%$ less likely with omicron than delta, but transmission a major concern
}

\section{Elisabeth Mahase}

Someone infected with the omicron variant of SARS-CoV-2 is estimated to be between $31 \%$ and $45 \%$ less likely to attend emergency care than if they had been infected with the delta variant and 50-70\% less likely to be admitted to hospital, analysis by the UK Health Security Agency has shown. ${ }^{1}$

But the agency said the findings, which exclude people with previous SARS-CoV-2 infection, are preliminary and highly uncertain because of the small numbers of hospital cases of omicron, an inability to effectively measure all previous infections, and the limited spread of omicron into older age groups.

It also emphasised that although a smaller proportion of people with omicron could end up in hospital than with previous variants, the actual number becoming seriously ill and needing hospital care could be huge, because of the variant's increased transmissibility.

The agency's chief executive, Jenny Harries, said, "Cases are currently very high in the UK, and even a relatively low proportion requiring hospitalisation could result in a significant number of people becoming seriously ill. The best way that you can protect yourself is to come forward for your first two doses of vaccine, or your booster jab, and do everything you can to stop onward transmission of the infection."

As at 20 December 132 people with confirmed omicron had been admitted to or transferred from hospital emergency departments. Notably, over $40 \%$ of hospital admissions were in London. Of the 132 patients, 17 had received a booster vaccine (three vaccine doses in total), 74 had received two doses, and 27 were not vaccinated. The vaccination status of six people was unknown, while eight had received a single dose. Within 28 days of an omicron diagnosis, 14 people were reported to have died, ranging in age from 52 to 96 years old.

The analysis, published on 23 December, also found that omicron was more likely than previous variants to reinfect people who have previously had covid-19, with $9.5 \%$ of those infected having a history of past infection. This figure is likely to be an underestimate because of some people being unaware that they had had asymptomatic prior infections, meaning they are not reported in the numbers.

Looking at vaccine efficacy, the report said that the data are continuing to show lower effectiveness against symptomatic omicron. Evidence indicates that although two doses of vaccine are less effective against omicron than against the previously dominant delta variant, a booster dose improves protection. But this extra protection may wane more rapidly against omicron than delta, being about 15-25\% lower from 10 weeks after the booster.
It's still too early to estimate vaccine effectiveness against hospital admissions, but the agency said that this was more likely to be sustained, particularly after a booster.

England's health and social care secretary, Sajid Javid, said, "Hospital admissions are increasing, and we cannot risk the NHS being overwhelmed. This is early stage analysis, and we continue to monitor the data hour by hour."

The agency's findings are consistent with three recent studies, not yet peer reviewed, from researchers in England, Scotland, and South Africa, which all concluded that omicron carried a lower risk of hospital admission than delta. ${ }^{2}$

\section{UK Health Security Agency. SARS-CoV-2 variants of concern and variants under investigation in England: technical briefing 33. Dec 2021. https://as- sets.publishing.service.gov.uk/government/uploads/system/uploads/attach- ment_data/file/1043680/technical-briefing-33.pdf. \\ 2 Christie B. Covid-19: Early studies give hope omicron is milder than other variants. BMJ 2021;375doi: 10.1136/bmi.n3144.}

This article is made freely available for use in accordance with BMJ's website terms and conditions for the duration of the covid-19 pandemic or until otherwise determined by BMJ. You may use, download and print the article for any lawful, non-commercial purpose (including text and data mining) provided that all copyright notices and trade marks are retained. 